This document is the accepted manuscript version of the following article:

Fumey, B., Baldini, L., \& Borgschulte, A. (2020). Water transport in aqueous

sodium hydroxide films for liquid sorption heat storage. Energy Technology,

2000187 (8 pp.). https://doi.org/10.1002/ente.202000187

\title{
Title: Water Transport in Aqueous Sodium Hydroxide Films for Liquid Sorption Heat Storage
}

Authors: B. Fumey*, L. Baldini and A. Borgschulte

\section{Affiliations:}

Benjamin Fumey, Dr. Luca Baldini

Urban Energy Systems Laboratory

Empa - Swiss Federal Laboratories for Materials Science and Technology

Überlandstrasse 129

8600 Dübendorf

Switzerland

E-mail: benjamin.fumey@empa.ch

Dr. Andreas Borgschulte

Advanced Analytical Technologies Laboratory

Empa - Swiss Federal Laboratories for Materials Science \& Technology

Überlandstrasse 129

8600 Dübendorf

Switzerland

\section{Keywords}

Absorption heat storage, Inter-seasonal heat storage, Raman spectroscopy, Mass transport analysis

\section{Conflict of Interest}

There is no conflict of interest.

Received: ((will be filled in by the editorial staff))

Revised: ((will be filled in by the editorial staff)) Published online: ((will be filled in by the editorial staff))

\begin{abstract}
Compact inter-seasonal thermal storage is a key enabler for renewable heating. A promising approach considered is the liquid absorption process, extended with absorbent and absorbate storage. In sorption heat storage, the conventional parameters; temperature gain and power density, governing the sorption heat pump process, are extended by the parameter energy density. This opens up new challenges for heat and mass exchanger design, demanding a detailed understanding of the fundamental mass transport process under technically relevant constraints. Towards this object, investigation in the water mass transport in static aqueous sodium hydroxide thin film at application specific temperature and pressure using temporally and spatially resolved Raman spectroscopy was performed. Based on the measured
\end{abstract}


concentration gradient in the film it was determined that the mass transport in the film and not the liquid gas interface is limiting.

\section{Introduction}

Bridging renewable energy supply and building heat demand requires inter-seasonal thermal energy storage. ${ }^{[1-4]}$ Liquid sorption heat storage is a propitious field of research towards this requisition, featuring loss-free storage over time, potentially high energy density and relatively low cost. ${ }^{[5-7]}$ The concept relies on a sorption heat pump extended with liquid sorbent and sorbate storage. Typical hygroscopic fluids studied are aqueous solutions of lithium bromide, lithium chloride, calcium chloride and sodium hydroxide, with sodium hydroxide showing superior potential in respect to high energy density and low material price. ${ }^{[8-11]}$ To date, no functional storage systems are available, past demonstrators have failed to reach anticipated and required temperature gain, power, and energy density. ${ }^{[12]}$ In sorption heat storage, static temperature gain, vapor pressure and concentration of the absorbent are interdependent and ultimately restricted by equilibrium thermodynamics, limiting maximum energy density. However, in light of the dynamic process, kinetic limitations govern, diminishing practicable storage capacity. Consequently, a fundamental limitation in liquid sorption storage performance is heat and mass exchange kinetics under application-specific conditions. In an approach to resolve this dependency, a vertically installed spiral finned tube counter-flow heat and mass exchanger was designed, in which the sorbent flows on the fin as a thin film in a downwards spiral motion. ${ }^{[13]}$ The design successfully enables improved concentration change in single pass by constant thin film flow and long absorbent to absorbate exposure time, increasing practicable energy density. To understand the mass transport in the thin film, and possibly ascertain methods to speed mass transport for increased volumetric specific power, detailed examination of the mass transport process in absorbent thin film under application condition was required. For this, a method 
with spatial and temporal resolution on the micrometer and minutes scale was necessary, compatible in harsh conditions, at water vapor pressures atmosphere of several mbar, elevated temperature and in the corrosive media aqueous sodium hydroxide.

An approach was found by means of depth profiling through confocal Raman spectroscopy. By this measuring method, it was found that under application-specific conditions mass transport of water in the sodium hydroxide film was rate-limiting. A diffusion parameter of water in aqueous sodium hydroxide under operating conditions was determined, which serves as a basis for further development of the sorption heat storage specific heat and mass exchanger designed.

\section{Discussion and Results}

The water vapor pressure on the aqueous sodium hydroxide solution is concentration and temperature dependent. An increase (decrease) in the water vapor pressure or a decrease (increase) in the solution temperature leads to absorption (desorption) of water, thus increasing (decreasing) water concentration at the solution's surface. The resulting chemical current of water molecules $j$ :

$j=\dot{M} \cdot A^{-1}\left[\mathrm{~mol} \cdot \mathrm{m}^{-2} \cdot \mathrm{s}^{-1}\right]$

where $\dot{M}$ is the molar mass flow and $A$ is the active area, going into (out of) the sorbent depends on the coupled mass transport of the gas phase, the uptake and release of sorbate at the gas-liquid interface and the mass transport of the liquid. The chemical current relates to the thermal power $P=\dot{q}$ of a specific heat and mass exchanger:

$P=\dot{q}=j \cdot A \cdot \Delta H[\mathrm{~W}]$

where $j$ is the chemical current, $A$ is the active area and $\Delta H$ is the enthalpy of absorption. Mass transport resistance in the gas phase can be neglected under the condition that only water vapor is present in a closed technical process. The question remains whether the kinetic barrier at the gas-liquid interface or the mass transport in the film is rate limiting. 
The description of the chemical current $j_{\text {int }}$ at the interface is complex; a simplified representation is the Hertz-Knudsen equation: ${ }^{[14,15]}$

$j_{\text {int }}=\left(M \cdot\left(2 \pi \cdot k_{B}\right)^{-1}\right)^{0.5} \cdot\left(\sigma_{d} \cdot p_{v} \cdot \theta_{L^{-0.5}}-\sigma_{a} \cdot p_{s} \cdot \theta_{V^{-}}^{-0.5}\right)\left[\mathrm{mol} \cdot \mathrm{m}^{-2} \cdot \mathrm{s}^{-1}\right]$

where, $M$ is the mass of the water molecule, $k_{B}$ is the Boltzmann constant, $\sigma_{d}$ and $\sigma_{a}$ are sorption coefficients, $p_{s}$ is the absorbent surface equilibrium (saturation) pressure, $p_{v}$ is the gas (sorption vapor) pressure, $\theta_{L}$ is the temperature of the liquid phase and $\theta_{V}$ is the temperature of the gas phase.

By assuming the various coefficients to be around one, and temperature equilibrium between the gas and liquid phases, the current depends on the concentration of the topmost layer $(\Rightarrow$ $p_{s}$ ), which increases with absorption (and vice versa). The concentration difference between the gas-liquid interface and deeper layers decays via the diffusion of water molecules away from (towards) the gas-liquid interface. Consequently, if the diffusion is rate-limiting, the interface layer will be in equilibrium with the gas phase, i.e. $j_{\text {int }}$ will be irrelevant (as $p_{v}=p_{s}$ ) and if the diffusion is fast, the interface is dominant. Diffusion is identified as rate limiting, if a temperature or concentration gradient $\left(\Delta X \cdot \Delta L^{-1}\right.$ and $\Delta \theta \cdot \Delta L^{-1}$, respectively) is measured:

$j_{\text {diff }}=-D \cdot \Delta X \cdot \Delta L^{-1}$

$\dot{q}=-\lambda \cdot \Delta \theta \cdot \Delta L^{-1}$

where $\lambda$ and $D$ are the thermal conductivity and mass diffusion coefficients respectively, $X$ is the concentration, $\theta$ is the temperature and $L$ is the film height. 


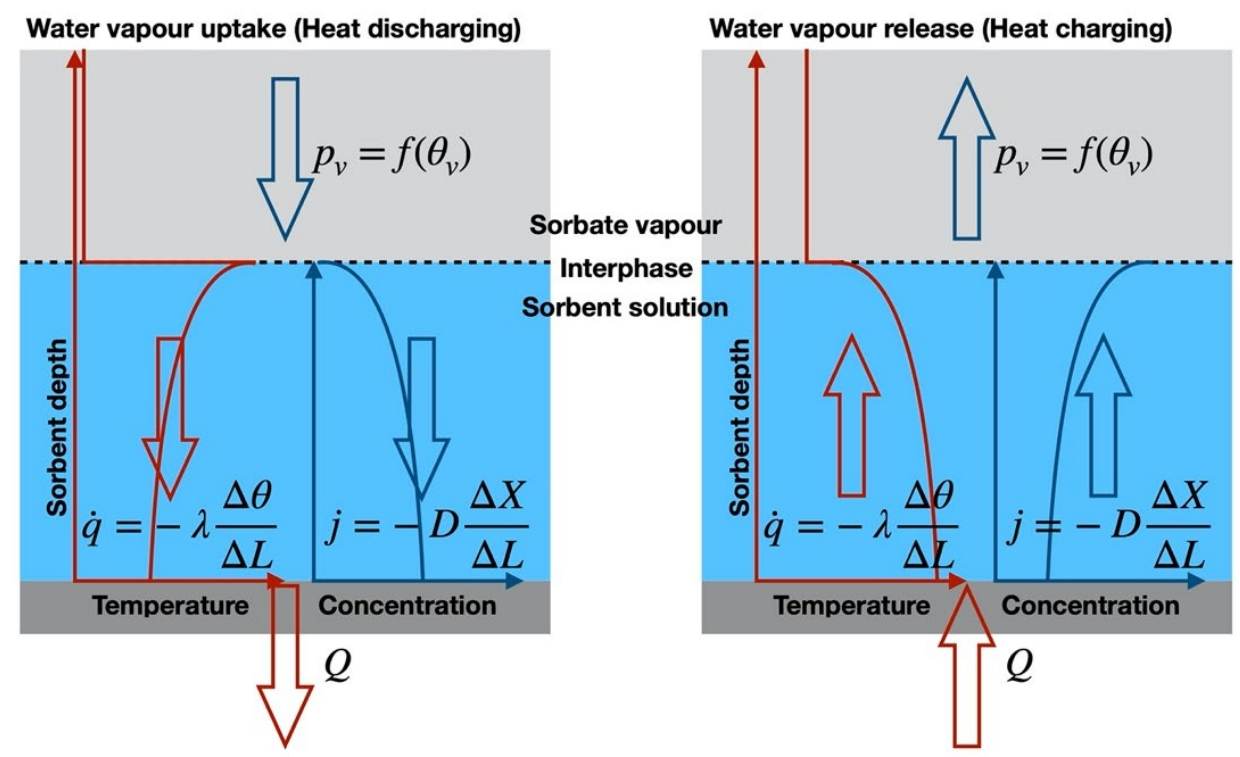

Figure 1. Illustration of the general behavior of the temperature and sorbent concentration in the sorbent film during water vapor uptake and release.

The generally recognized behaviors of the temperature and concentration are illustrated in figure 1. Both thermal and chemical diffusion leads to exponential-like gradients. The discontinuities are a consequence of the interface phenomenon (Herz-Knudsen equation, equation 3). The temperature gradient in the film was found to be relatively small. Combining equations 4 and 5 yields:

$\Delta \theta \cdot \Delta L^{-1}=D \cdot \lambda^{-1} \cdot \Delta H \cdot \Delta X \cdot \Delta L^{-1}$

$\rightarrow \Delta \theta \approx 2 \cdot 10^{-11} \mathrm{~m}^{2} \mathrm{~s}^{-1} \cdot 5 \cdot 10^{4} \mathrm{~J} \mathrm{~mol}^{-1} \cdot\left(0.65 \mathrm{~J}^{-1} \mathrm{~K}^{-1} \mathrm{~m}^{-1}\right)^{-1} \cdot 10^{4} \mathrm{~mol} \mathrm{~m} \mathrm{~m}^{-3}=0.015 \mathrm{~K}$.

Assuming a maximum concentration difference of $\Delta X=10 \mathrm{~mol} L^{-1}(50 \mathrm{wt} \%$ down to $30 \mathrm{wt} \%)$ over the full film thickness. An assumption well-fitting to the application.

Practically all material parameters depend on concentration and temperature and are hence only partially known, complete modeling is thus associated with a rather large uncertainty value. Consequently, a more pragmatic approach is followed. Knowing the experimental results of the measurements, it was possible to simplify the modeling based on the following consideration. If a strong chemical gradient is measured in the liquid phase, this gradient would be rate limiting, implying that the gas-liquid interface shown in equation 3 and the heat 
transport, see equation 5 are less relevant. In this case, the empirically determined diffusion coefficients must match the corresponding literature values.

A series of measurements on the time-resolved concentration changes during absorption and desorption at varying focal points of the sorbent solution film were derived, as presented in the experimental section. Figure 2 shows an example of measurement sequence 4 (see Table 1) with the individual curves measured at a defined distance from the film bottom. The dot sequence measured at $100 \mu \mathrm{m}$ (violet) was regarded as the concentration at the film bottom. The determination of the concentration at the gas-liquid interface was derived by considering the intensity of the Raman scattered light, indicated by the size of the corresponding dots. The intensities of the measurements near the film bottom did not substantially change but those at $200 \mu \mathrm{m}$ and above did. Abruptly, at measurement 13, the intensities of the measurement series at $200 \mu \mathrm{m}$ increased. Here, the focal point dipped into the solution because the film grew due to the water uptake. The same situation occurred two measurements (30s) later for the measurement series at $250 \mu \mathrm{m}$ and finally at $300 \mu \mathrm{m}$. The concentration at the gas-liquid interface could then be reconstructed from the entry points, shown by the gray line in figure 2 . As the droplet height decreased during desorption, the same process occurred in reverse. Through this analysis, the concentration changes based on time $\partial X \cdot \partial T^{-1}$ and the concentration gradient $\partial X \cdot \partial L^{-1}$ in the film were derived. 


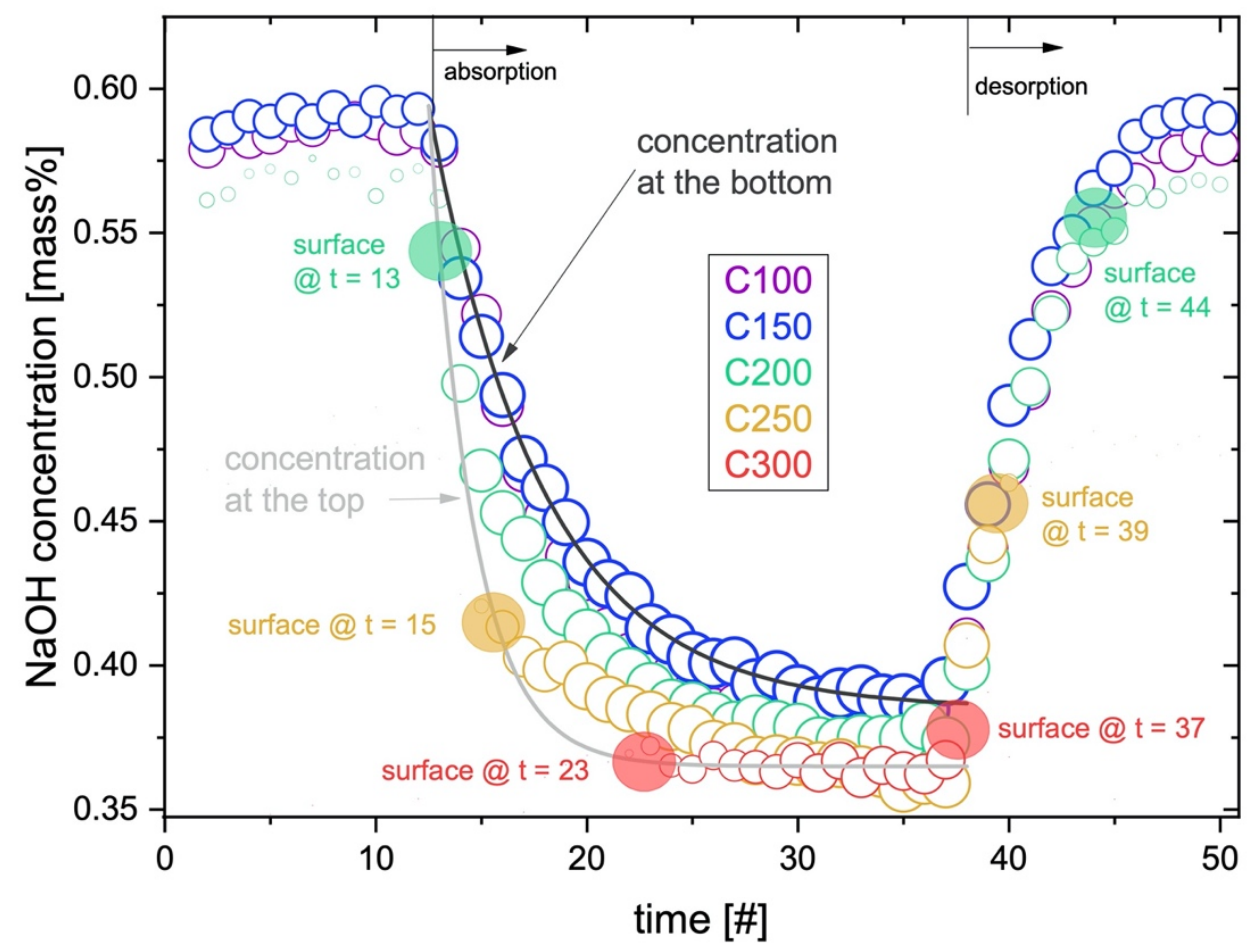

Figure 2. Spatially and temporally resolved sodium hydroxide concentrations measured operando during water absorption and desorption at a temperature of $35^{\circ} \mathrm{C}$ and $55^{\circ} \mathrm{C}$, respectively, and a constant vapor pressure of $8.7 \mathrm{mbar}$. The data shown here was derived from measurement sequence 4 (see Table 1). The color code indicates the position of the focal point, i.e., the measurement with respect to the thin film bottom. As the thin film thickness changed upon water uptake or release, the higher focal points dipped into or dropped out of the film. To highlight this behavior, the total intensity of the Raman light is shown as the diameter of the data points. The grey line is the extrapolated surface concentration as a function of time.

The aim of this analysis was to determine whether the water uptake and release in sodium hydroxide is diffusion or interface controlled. The exponential temporal decay and growth of the concentration in the film, shown in figure 2, were strong indications in support of diffusion limitation. However, specific surface barriers, as discussed in the introduction, can also result in such behavior. To substantiate the rate-limiting aspect of diffusion, the link between the concentration gradient in the film $\partial \mathrm{X} \cdot \partial \mathrm{T}^{-1}$ and the water uptake/release rate $\boldsymbol{F}$ of the film had to be proven. The water uptake/release rate was empirically derived from the time derivative of the total amount diffused into the film: 
$F \propto \frac{d}{d t}\left(\frac{M_{t}}{M_{0}}\right), \frac{M_{t}}{M_{0}} \propto \sum_{i} \frac{X_{i} I_{i}}{I_{i}}$

where $M_{0}$ is the total substance amount diffused at time $0, M_{t}$ is the total substance amount diffused at time $t, X_{i}$ is the concentration, $I$ is the Raman intensity and $i$ is the Raman spectral measurement number.

On the other hand, the mass transport rate into a film can be calculated from the concentration gradient, if diffusion is rate limiting.

$F=-D\left(\partial X \cdot \partial L^{-1}\right)_{t o p}$

where $D$ is the diffusion coefficient and $\left(\partial X \cdot \partial L^{-1}\right)_{t o p}$ is the spatial concentration gradient to the film surface.

For diffusion into a plane sheet with the usual boundary conditions, the normalized uptake is: ${ }^{[28]}$

$\frac{M_{t}}{M_{0}}=1-\frac{8}{\pi^{2}} \sum_{n=0}^{\infty} \frac{1}{(2 n+1)^{2}} \exp \left\{-\frac{D(2 n+1)^{2} \pi^{2} t}{L^{2}}\right\}$

where $L$ is the film height.

Equation 9 is a solution to Fick's 2nd law:

$\left(\partial X \cdot \partial T^{-1}\right)=D\left(\partial^{2} X \cdot \partial L^{-2}\right)$

Whereby, this function is solely determined by the parameter $D \cdot L^{-2}$.

A fit thereof gave $D \cdot L^{-2}=0.0013 \mathrm{~s}^{-1}$, from which a diffusion coefficient of $D=2 \cdot 10^{-10} \mathrm{~m}^{2} \mathrm{~s}^{-1}$ was derived by assuming a constant average film thickness of $250 \mu \mathrm{m}$. Once obtained, this parameter was used to simulate the concentration gradient:

$\left(\frac{\partial X}{\partial L}\right)_{t o p}=-\frac{F}{D} \propto \sum_{n=0}^{\infty} \exp \left\{\frac{D}{l^{2}}(2 n+1)^{2} \pi^{2} t\right\}$

The obtained agreement between the simulated and measured data was experimental proof of diffusion being the rate-limiting step during the water uptake and release into aqueous sodium hydroxide, as shown in the bottom panel of figure 3. 


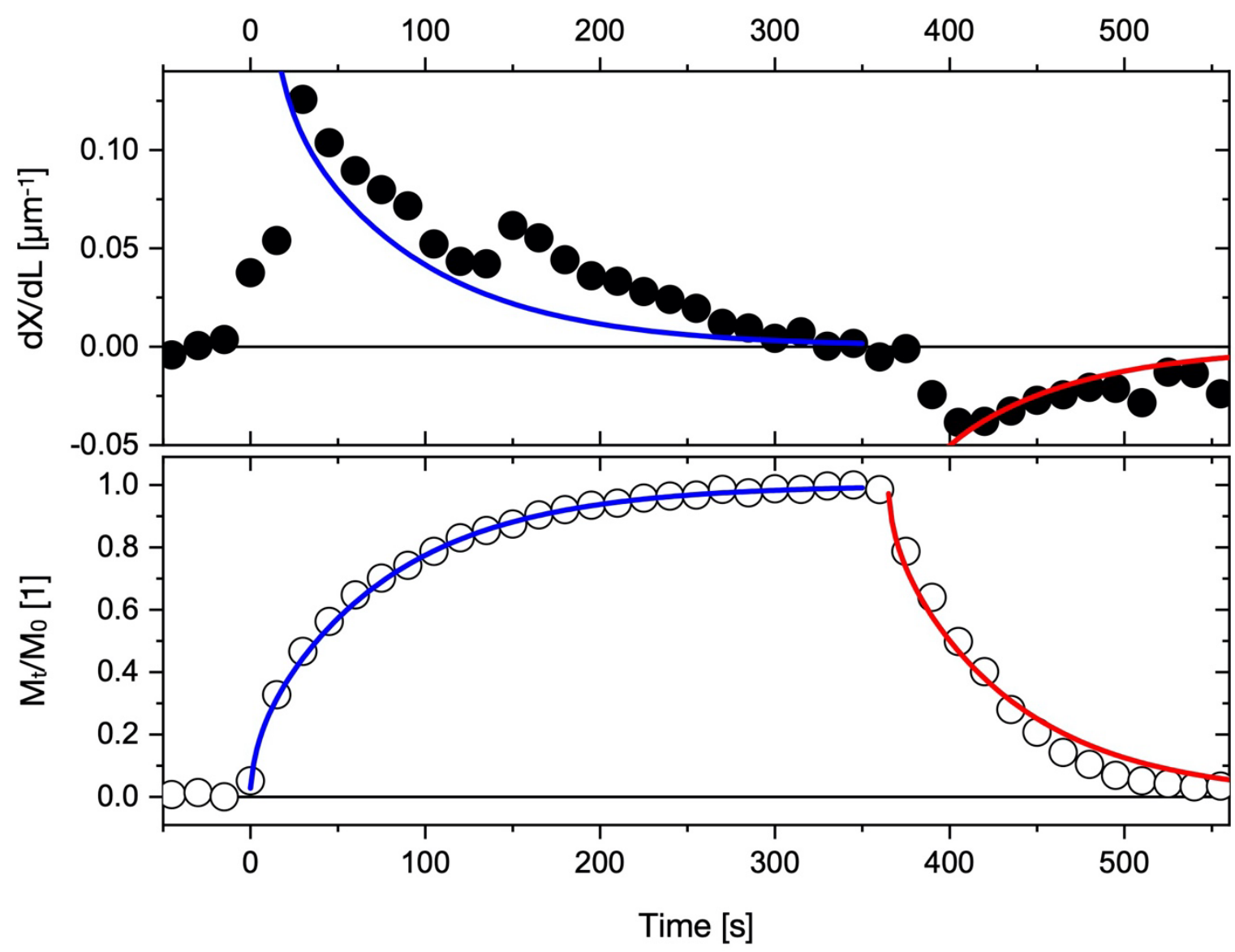

Figure 3. The top panel shows the concentration gradient averaged over the full thin film thickness (dots). The line depicts the simulated concentration gradient at the surface, using equation 11 with $D \cdot L^{-2}$ as the fit parameter, derived from the fit of the time evolution of the total uptake to equation 9. Bottom panel: The normalized total amount of water taken versus time derived from the data shown in figure 2. The lines fit with the experimental data points.

At this point, there were several experimental uncertainties that must be noted. The measured gradient, as shown in the top panel of figure 3, was consistently average throughout the film, whereby the film thickness changed during sorption. The calculated gradient, on the other hand, was an infinitesimal number defined at the sorbent surface. This was also the reason for the additional peak at time $120 \mathrm{~s}$. Nevertheless, this did not affect the qualitative statement, showing the gradient curve expected when mass diffusion is rate limiting. To derive the quantitative diffusion parameters from the measurements with various thermodynamic conditions, as per the measurement sequences shown in table 1, the analysis of equation 11 was simplified by determining the time of the half-uptake $\tau^{[16]}$ 
where $L$ is the average film depth (see Table 1).

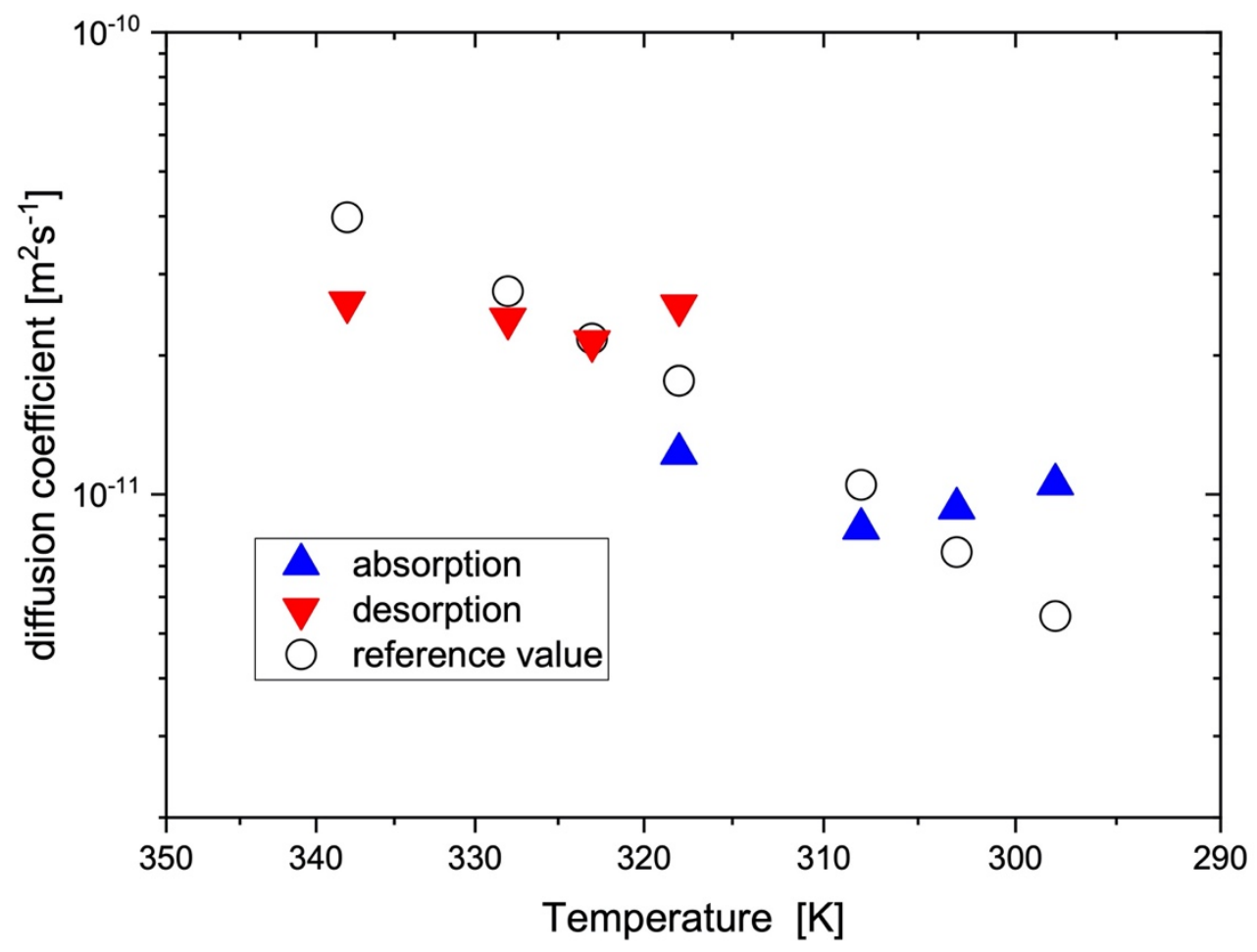

Figure 4. The diffusion coefficient of water in aqueous sodium hydroxide at various temperatures. The measured results were based on the half-time measurements calculated according to equation 12 , while the reference values were calculated from the viscosity data according to equation 13 .

The concentration curve at the $100 \mu \mathrm{m}$ focal point was considered as the mean value. In figure 4, the average diffusion coefficients obtained from the four varying vapor pressure settings (see Table 1) are shown, in comparison to the calculated values based on the dynamic viscosity of aqueous sodium hydroxide: ${ }^{[17]}$

$D=k_{B} \cdot \theta \cdot(b \cdot \pi \cdot \eta \cdot r)^{-1}$

where $k_{B}$ is $1.3806 \cdot 10^{22} \mathrm{JK}^{-1}, \theta$ is the temperature of the sorbent during absorption or desorption, $b$ is a correction factor take to be $4, \eta$ is the dynamic viscosity (average dynamic viscosity between $55 \mathrm{wt} \%$ down to $40 \mathrm{wt} \%$.) and $r$ is the molecular radius of $\mathrm{H}_{2} \mathrm{O}$ taken to be $1.375 \cdot 10^{-10} \mathrm{~m}$. 
The reference and measured values were very closely fitting. The slight diversion was considered to be due to measurement uncertainties. The trend shows that the values at the $0^{\circ} \mathrm{C}$ evaporating temperature were slightly greater than the reference, and the values at the $20^{\circ} \mathrm{C}$ evaporating temperature were lower.

In phase transitions of pure substances, such as the evaporation of water, where no substance redistribution takes place in the bulk, the interface between the gas and liquid is a clear determinant for the mass transfer rate. ${ }^{[18]}$ On the other hand, in pools of sorbents, such as aqueous sodium hydroxide, it is not surprising that diffusion is the mass transfer rate limiting step. Nevertheless, when switching to thin films as followed in the spiral finned tube heat and mass exchanger, there was no clear delineation of the surface layer from the bulk such that no obvious conclusions could be drawn from the previous examples.

A model commonly adopted to describe the physical absorption of a gas into a liquid is the laminar film model by Whitman. ${ }^{[19]}$ As noted by Danckwerts and Kennedy, this model assumes a laminar gas-liquid interface layer as the predominant constriction to mass transfer the gas into the liquid phase, even under bulk turbulence. ${ }^{[20]}$ Tsai and Perez-Blanco relied on similar assumptions that the surface layer close to the phase boundary determines the mass transfer rate, and consequently, surface-to-bulk mixing is required to increase the transfer rates beyond those achieved by molecular diffusion. ${ }^{[21]}$ Based on this, it was initially consented that surface breakup was needed to increase the mass transfer rate on the highly laminar creeping flow present on the technical spiral finned tube. ${ }^{[13]}$ For this reason, experiments with cutouts on the heat exchanger were performed, affecting droplet formation and impingement into the liquid sorbent film to promote surface breakup. While the exact effect of this droplet impingement under varying parameters is yet to be studied and fully understood, there was no confirmation of a mass transfer rate increase in our assessment. The mere surface breakup achieved by the impinging droplet did not appear to be effective. This may be due to the insufficient momentum of the impinging droplet or because not the surface 
layer but the entire film is determinant for the mass transfer, hinting towards the importance of molecular diffusion across the entire liquid film.

With the reported novel approach to in-situ probing the reversible water sorption in thin aqueous sodium hydroxide films using Raman spectroscopy, it was possible to clearly show that the rate-limiting step in this process is the mass diffusion from the gas-liquid interface to the film body. Although many studies on water absorption in liquid salt solutions associated with large heat gain, as is the case here, have been carried out in the past, this is the first time that the transient concentration gradients of a thin film sorption process with high temporal and spatial resolutions in application relevant conditions have been measured. ${ }^{[22-31]}$ In the past, studies relied on measuring sorption into bulk sorbents at a constant concentration and temperature using setups such as stirred cell reactors and pool absorbers or absorption in wetted-wall columns and disk columns with turbulence, thus making the evaluation of the individual transport barriers or spatial-resolved measurements unattainable.

The spatial resolution of several $10 \mu \mathrm{m}$ and temporal resolution of $15 \mathrm{~s}$ were attributed to the Raman apparatus employed. With this limited resolution, which may be improved using advanced Raman microscopes, and related optical methods such as sum frequency generation and high-pressure photoemission, the existence of a rate-limiting gas-liquid interface barrier could not be rule out. ${ }^{[32,33]}$ However, the film thickness used is alike to that present in the technical spiral finned heat and mass exchanger. In this design, the heat transport fluid flows through the tube from bottom to top, and the aqueous sodium hydroxide flows by gravitational force from top to bottom on the external spiral fin. Long sorbent exposure times for improved mass and heat flux was so made possible.

The identification of a diffusional gradient with diffusion parameters similar to the intrinsic values (see figure 4), was evidence that the influence of the gas-liquid interface barrier was secondary and that diffusion was generally rate limiting even under thin film conditions. The influence of the gas-liquid interface barrier is expected to increase with forced mixing in the 
bulk of the film as the hindrance by diffusion is reduced ${ }^{[34]}$ However, due to the high viscosity of the sorbent and low film height, the technical realization is impracticable and diffusion remains the main restriction to high mass uptake kinetics.

The evidence for classical diffusion as a rate-limiting step simplifies the realistic modeling of thin film mass transfers in technical heat and mass exchangers since the slow diffusion of water in aqueous sodium hydroxide is well known. Previously published model calculations can be based on. Nevertheless, models for mass transfer from the gas phase to liquid phase, such as the stagnant film model, the penetration model and the film-penetration model, all assume the presence of a well-mixed liquid bulk. ${ }^{[19,35,36]}$ In our thin-film application, the issue concerning the absence of a constant concentration and temperature bulk is that the effective driving force for mass transfer is reduced due to the reduction of the sorbent concentration in the solution contacting the solid interface. This decreases the mass transfer flux and thus also reduces the average flux, as seen in the top panel of figure 3 . Therefore, these models may lead to overly optimistic values for the mass transfer flux found in technical heat and mass exchangers.

The techno-economic viability, volume and cost of the liquid sorption heat storage strongly depends on the heat and mass exchanger temperature gain, power and system energy density. High energy density requires a large sorbate swing rate, whereby the increase in the sorbate concentration decreases mass flux and thus power, as shown in figure 2. In the technical process, there are two concentration gradient levels to be considered: across the film height (distance) and along the heat and mass exchanger (time). The first gradient can be countered by turbulence, in maximum reducing the concentration gradient in the film to a mean level and substantially reducing the mass transport resistance into the film. The second gradient along the spiral finned tub, can nevertheless not be removed. This gradient is also seen in figure 2 along the time axis, showing the consequent reduction of the driving force for the 
mass transfer. It must thus be recognized that there are fundamental restrictions to the augmentation of power in heat and mass exchangers for sorption heat storage.

In comparing the diffusion coefficient of the heat and mass exchanger, calculated according to equation 2 and 4, to the measured values in figure 4, it was recognized that the heat and mass exchanger, having a diffusion coefficient of $7.5 \times 10^{-9} \mathrm{~m}^{2 \cdot} \mathrm{s}^{-1}$ was a good order of magnitude higher. This suggested that even though a slowly creeping film flow was visually detected, some mixing must have been involved, possibly stemming from a combination of varying flow distances due to the spiral flow as well as the varying viscosities due to the concentration gradient present.

\section{Conclusion and Outlook}

In the reported work, the water sorption process in static aqueous sodium hydroxide thin films under technically relevant conditions using temporally and spatially resolved Raman spectroscopy was followed. The measured transient concentration gradient was evidence that water sorption kinetics is limited by diffusional mass transport. It was found that in the technical heat and mass exchanger design for sorption heat storage, bulk mixing and not gasliquid interface break up was required. For the technical application, further work is now necessary to acquire an understanding of the flow regime along the vertically positioned counter-flow spiral finned tube heat and mass exchanger to increase mixing and thus improve mass flux kinetics for enhanced power and energy performance. In order to evaluate concentration gradient and bulk mixing in operando, the use of Raman based hyperspectral imaging will be sought, potentially enabling direct 2D Raman intensity mapping by which concentration distribution maps can be derived. In this way, complex concentration gradient movement in the flowing film and along the spiral finned tube can be determined and turbulence through concentration movement can be detected. 
The novel approach to highly resolved mass transport analysis described in this work, can further be applied to other fields of study, such as uptake of $\mathrm{CO}_{2}$ in water films potentially providing additional understanding of the process in the gas to liquid interphase.

\section{Experimental Section}

The spatial and temporal probing of the concentration gradient of a thin aqueous sodium hydroxide film (approximately $200 \mu \mathrm{m}$ ) serving as sorbent, requires a compound selective method compatible with the corrosive environment as well as the required spatial and temporal resolution. Since water, aqueous sodium hydroxide and glass, employed as an atmospheric barrier, are transparent to visible light, Raman spectroscopy was found to be a fitting approach. Raman spectroscopy is commonly used to provide structural fingerprints by which molecules can be identified. For liquids and gases, the corresponding peak intensities (Raman traces) are related to the number of scattering molecules. ${ }^{[37]}$ Raman scattering or inelastic photon scattering is a scattering technique. The Raman signal stems from the laser focal point with a resolution of around $5 \mu \mathrm{m}$. The spatial resolution is limited due to the relatively large working distance and beam path traveling through a window. With the appropriate mechano-optics (mechanical xyz-stage integrated into a confocal microscope), the Raman traces can be assembled to provide a 3D image of the voxel under investigation.

Raman spectroscopy has been employed for the investigation of microscopic phenomena such

as diffusion. ${ }^{[38-40]}$ In this work, the method was adapted to obtain time-resolved measurements of sodium hydroxide concentrations at varying film depths in the process of absorption and desorption, with a time resolution of minutes and a practical spatial resolution of several ten micrometers using a Bruker Raman SENTERRA Spectrometer (532 nm) with a home-built Raman sorption analysis cell.

To realistically mimic the water exchange of the gas-liquid interface in a technical heat and mass exchanger, an optical cell was designed, in which the sorption and desorption into a liquid reservoir of aqueous sodium hydroxide with a water vapor atmosphere and elevated 
temperatures was operable. The assumptions were as follows: The sorbent is a thin film and immobile, so turbulences in the liquid must be excluded, and changes in the sorbent solution's temperature causing absorption and desorption occur rapidly. The first assumption was justified as the viscosity of the aqueous sodium hydroxide solution was high, the film height was low and boiling in desorption was prevented. ${ }^{[41]}$ The second assumption was considered due to the thin sorbent film having higher thermal conductivity than water and the powerful Peltier element involved in heating and cooling. ${ }^{[42]}$ Contrarily, in the technical heat and mass exchanger, the sorbent is not exposed to step-wise temperature changes but to a gradual change. Consequently, the technical absorption and desorption processes are expected to be slower.

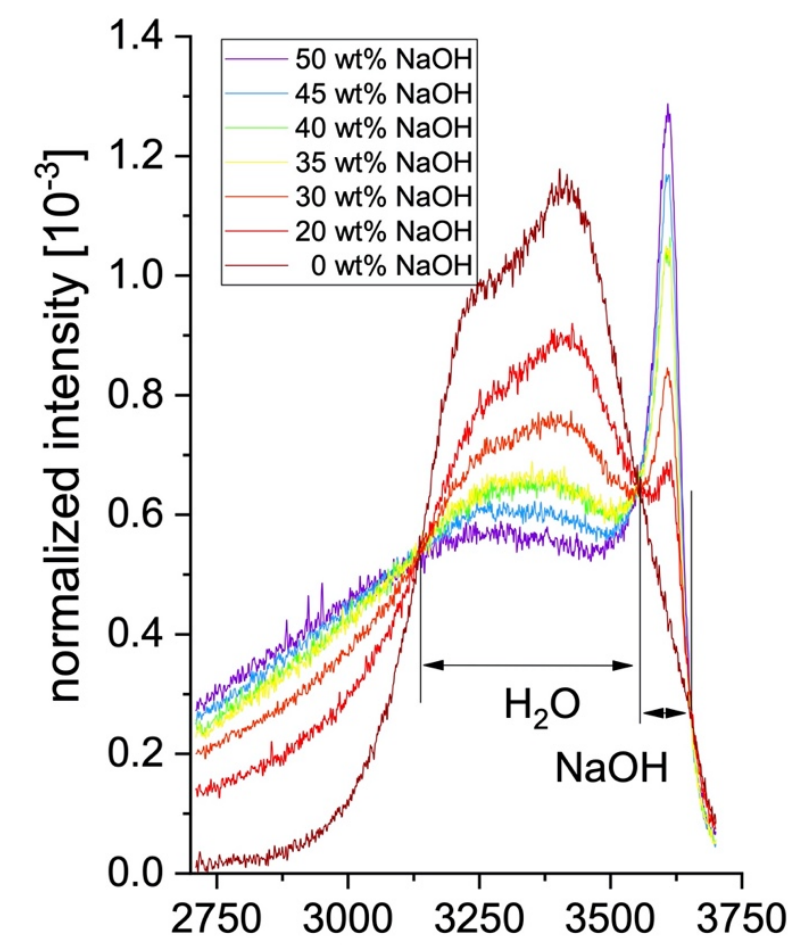

(a)

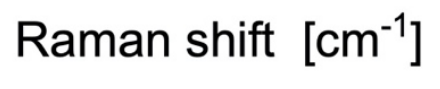

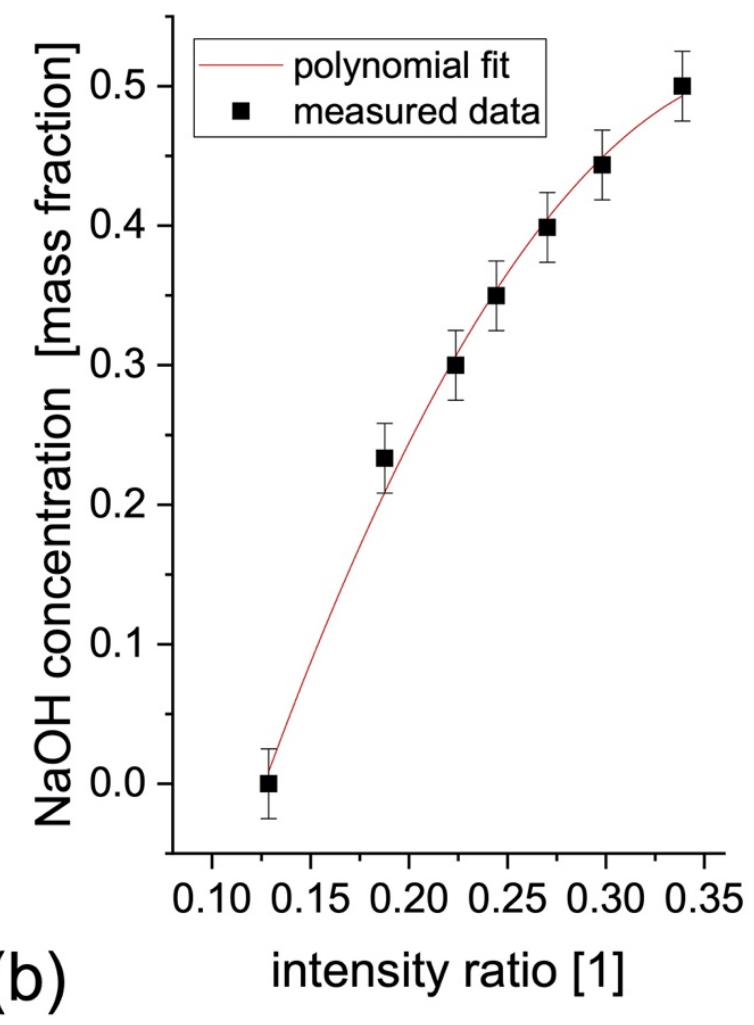

Figure 5. (a) Total-intensity normalized Raman spectra of aqueous sodium hydroxide solutions at various concentrations; the lines indicate the isosbestic points which define the integration boundaries for quantification. (b) Calibration curve to determine the spatially resolved sodium hydroxide concentration used throughout the paper, derived from the intensity ratio of the two integration areas in (a). 
For the investigation, a test cell was constructed, consisted of a stainless-steel body with a center-drilled compartment and a $60 \mathrm{~mm}$ by $2 \mathrm{~mm}$ round Suprasil ${ }^{\circledR}$ glass covering window, as shown in figure 5a. A Peltier element for heating and cooling was placed inside the chamber with a stainless-steel plate as the sample holder. The setup was connected to a water vapor source and vacuum pump for pressure regulation. By moving the xyz-table towards the Raman microscope objective, the Raman focal point was moved into the solution.

The molecular vibrations used were $\mathrm{OH}^{-}$stretching vibrations occurring in both water and sodium hydroxide around $3600 \mathrm{~cm}^{-1}$, as shown in figure $5 \mathrm{a}$. As the solvated $\mathrm{OH}^{-}$ion is chemically different from the $\mathrm{OH}$ in water, $\mathrm{OH}^{-}$ions and the $\mathrm{OH}$ in water have slightly different Raman shifts. However, the local environments of the $\mathrm{OH}^{-}$ions and water molecules depend on the overall concentration. ${ }^{[43]}$ In the solution, the ions are surrounded by water molecules forming a hydrate shell (transient $\left.\left(\mathrm{H}_{3} \mathrm{O}_{2}\right)-\right)$. At low sodium hydroxide concentrations, the water molecules of these hydrate shell molecules are well separated by 'normal' statistically oriented water molecules, which constitute the majority of the water molecules. With increasing concentrations, these water molecules decrease in number until the liquid solidifies. The increasing correlation of the water molecules is reflected by Raman spectra; particularly, the water $\mathrm{OH}$ peak broadens and shifts with concentration. ${ }^{[43,44]}$ If the spectra are normalized, we can define isosbestic points, i.e., points where the normalized intensity does not change across various concentrations, see figure $5 \mathrm{a}$. This circumvents difficulties occurring with fitting the strongly overlapping peaks, as proposed by Shaffer et al. ${ }^{[44]}$ Still, the non-linear behavior is obvious, originating from the above-discussed dynamics of water in aqueous sodium hydroxide. The correlation between the given concentration and the normalized intensity ratios derived from the areas serves as the calibration, shown in figure $5 b$.

During measurement, an aqueous sodium hydroxide droplet was placed on the sample holder situated on top of the Peltier element and slightly smudged to create a thin film. All non- 
condensing gases were removed by applying a vacuum. Time was given for the sorbent droplet to reach the thermodynamic equilibrium of the vapor atmosphere determined by the aqueous sodium hydroxide film temperature, which was set by the Peltier element and the water vapor pressure. The challenge of the measurement lay in the fact that the position of the gas-liquid interface was not known a priori, and it changed upon absorption and desorption, a moving target. This was due to the uptake and release of water as illustrated in figure $5 \mathrm{~b}{ }^{[45]}$ Therefore, continuous Raman measurements were performed at a defined distance from the focal point to the bottom of the film (the sample holder) through a full cycle, incorporating a temperature change from high to low in absorption and low to high in desorption. One measurement succession, a complete absorption and desorption cycle, consisted of 49 Raman spectral measurements at a single $\mathrm{z}$-axis position. The time resolution was $10 \mathrm{~s}$. A measurement sequence, in turn, consisted of six successions with $\Delta z=50 \mu \mathrm{m}$. Due to the changing film thickness during the uptake and release of water, the z-position with respect to the gas-liquid interface was evaluated retrospectively, as shown in figure 6 . The Raman signal markedly changed when the focal point moved out of or into the liquid. Table 1 shows the typical measurement parameters of 12 measurement sequences performed under varying conditions with the same aqueous sodium hydroxide droplet.

(a)

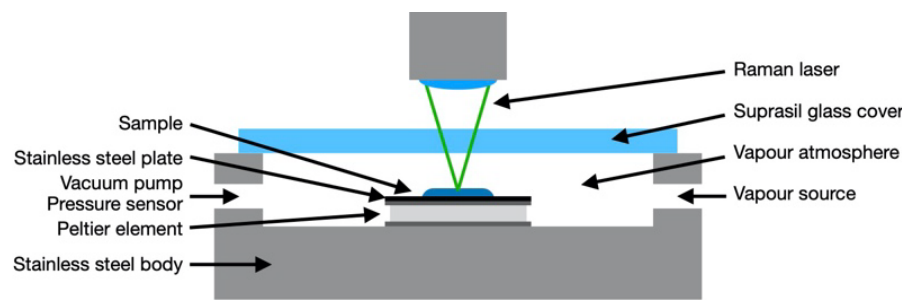

(b)

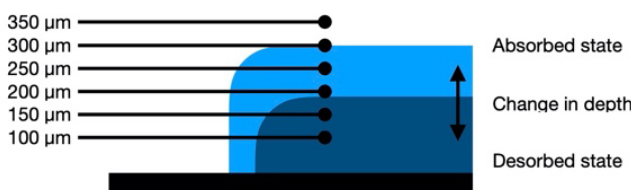

Figure 6. Test cell setup. (a) Illustration of the setup with the Peltier cooler and heater, stainless-steel plate and sample. (b) Illustration of the Raman depth profiling upon moving the focal point into or out of the aqueous sodium hydroxide film.

Table 1. Measurement parameters.

\begin{tabular}{lccccccc}
\hline $\begin{array}{l}\text { Measurement } \\
\text { sequence }\end{array}$ & $\theta_{\mathrm{ab}}\left[{ }^{\circ} \mathrm{C}\right]$ & $\theta_{\mathrm{de}}\left[{ }^{\circ} \mathrm{C}\right]$ & $\theta_{\text {vap }}\left[{ }^{\circ} \mathrm{C}\right]$ & $\mathrm{p}_{\mathrm{vap}}[\mathrm{mbar}]$ & $\mathrm{L}_{\min }[\mu \mathrm{m}]$ & $\mathrm{L}_{\text {max }}[\mu \mathrm{m}]$ & $\mathrm{L}_{\text {avg }}[\mu \mathrm{m}]$ \\
\hline 1 & 25 & 45 & 0 & 6.5 & 225 & 375 & 300 \\
2 & 25 & 45 & 0 & 6.5 & 200 & 300 & 250
\end{tabular}




\begin{tabular}{lllllllll}
3 & 30 & 50 & 5 & 8.7 & 175 & 275 & 225 \\
4 & 30 & 50 & 5 & 8.7 & 175 & 250 & 213 \\
5 & 35 & 55 & 10 & 12.2 & 150 & 250 & 200 \\
6 & 35 & 55 & 10 & 12.2 & 150 & 250 & 200 \\
7 & 45 & 65 & 20 & 23.3 & 150 & 225 & 188 \\
8 & 45 & 65 & 20 & 23.3 & 150 & 225 & 188 \\
9 & 45 & 65 & 20 & 23.3 & 125 & 200 & 163 \\
10 & 35 & 55 & 10 & 12.2 & 125 & 200 & 163 \\
11 & 30 & 50 & 5 & 8.7 & 100 & 175 & 138 \\
\hline
\end{tabular}

\section{Acknowledgements}

This research work was financially supported by the Swiss Innovation Agency Innosuisse grant Nr. 1155002545 and is part of the Swiss Competence Centre for Energy Research SCCER HaE. Supplementary funding was received from the Swiss Federal Office of Energy SFOE grant Nr. SI/501605-01 in the frame of the IEA SHC Task 58/ECES Annex 33 participation.

\section{References}

[1] J. C. Hadorn, Thermal energy storage for solar and low energy buildings - State of the art, ISBN: 84-8409-877-X,

[2] S. K. Shah, L. Aye, B. Rismanchi,Renew. Sust. Energ. Rev. 2018, 97.

[3] P. Pinel, C. A. Cruickshank, I. Beausoleil-Morrison, A. Wills, Renew. Sust. Energ. Rev. 2011, 15, 7.

[4] A. Mehari, Z.Y. Xu, R.Z. Wang, Energy Convers. Manag. 2020, 206.

[5] K. E. N'Tsoukpoe, H.Liu, N. Le Pierrès, L. Luo, Renew. Sust. Energ. Rev. 2009, 13.

[6] P. Tatsidjodoung, N. Le Pierrès, L. Luo, Renew. Sust. Energ. Rev. 2013, 18.

[7] X. Zhang, M. Li, W. Shi, B. Wang, X. Li, Energy Convers. Manag. 2014, 85.

[8] K. E.N’Tsoukpoe, N. Le Pierrès, L. Luo, Energy 2013, 53.

[9] C. Bales, Proceedings of DANVAK seminar, 2006.

[10] J. A. Quinnell, J. H. Davidson, J. Burch, Proceedings of ASME, 2010.

[11] R. Weber, V. Dorer, Vacuum 2008, 82, 7. 
[12] K. E. N’Tsoukpoe, N. Le Pierrès, L. Luo, Energy Procedia 2012, 30.

[13] B. Fumey, R. Weber, L. Baldini, Appl. Energy 2017, 200.

[14] V. V. Zhakhovsky, A. P. Kryukov, V. Y. Levashov, I. N. Shishkova, S. I. Anisimov, PNAS 2019, 116.

[15] A. H. Persad, C. A. Ward, Chem. Rev. 2016, 116.x

[16] Crank J. The Mathematics of Diffusion. ISBN 9780198534112

[17] J. Olsson, Å. Jernqvist, G. Aly, Int J Thermophys. 1997, 18.

[18] E. Y. Gatapova, I. A. Graur, O. A. Kabov, V. M. Aniskin, M. A. Filipenko, F.

Sharipov, L. Tadrist, Int. J. Heat Mass Transf. 2017, 104.

[19] W. G. Whitman, Chem. Met. Eng. 1923; 29.

[20] P. V. Danckwerts, A.M. Kennedy, Chem Eng Res Des. 1954; 75: S101-4.

[21] B. B.Tsai, H. Perez-Blanco, Int. J. Heat Mass Transf. 1998, 41.

[22] S. H. Chiang, H. L. Toor, AIChE 1964, 10.

[23] N. I. Grigoryeva, V. E. Nakoryakov, J Eng. Phys. 1977, 33.

[24] G. Grossman, Int. J. Heat Mass Transfer 1983, 26.

[25] N. Brauner, D. M. Maron, H. Meyerson, Int. J. Heat Mass Transf. 1989, 32.

[26] J. W. Andberg, G. C. Vliet, ASHRAE Trans 1987, 93.

[27] M. L. Yüksel, E. U. Schlündler, Chem Eng Process 1987, 22.

[28] N. Kawae, T. Shigechi, K. Kanemaru, T. Yamada, Heat Transf. Jpn. 1989; 8.

[29] B. J. C. VanDer Wekken, R. H. Wassenaar, Int J Refrig 1988, 11.

[30] N. Brauner, Int J Heat Mass Transf. 1991, 34.

[31] G. A. Ibrahim, G. A. Vinnicombe, Int J Heat Mass Transf. 1993, 36.

[32] T. L. Tarbuck, S. T. Ota, G. L. Richmond, J. Am. Chem. Soc. 2006, 128.

[33] R. K. Lam, J. W. Smith, A. M. Rizzuto, O. Karslığlu, H. Bluhm, R. J. Saykally, J.

Chem. Phys. 2017, 146.

[34] P. V. Danckwerts, Trans. Faraday Soc. 1951, 47. 
[35] R. Higbie, AIChE 1935, 31.

[36] H. L. Toor, J. M. Marchello, AlChE 1958; 4.

[37] F. Li, Z. Li, S. Wang, S. Li, Z. Men, S. Ouyang, C. Sun, Spectrochim. Acta A 2017 , 183.

[38] A. Borgschulte, R. Gremaud, Z. Łodziana, A. Züttel, Phys. Chem. Chem. Phys., 2010, 12.

[39] A. Borgschulte, R. Delmelle, R. B. Duarte, A. Heel, P. Boillatc, E. Lehmannc, Phys. Chem. Chem. Phys., 2016, 18.

[40] C. Kurt, J. Bittner, Sodium Hydroxide, in Ullmann's Encyclopedia of Industrial Chemistry. 2000, Wiley-VCH Verlag GmbH \& Co. KGaA.

[41] Caustic Soda - SOLVAY Technical and Engineering Service, Bulletin Nº 6 (1967)

[42] A. A. Alexandrov, 14th International Conference on the Properties of Water and Steam in Kyoto 2005.

[43] G. E. Walrafen, R. T. Douglas, J. Chem. Phys. 2006; 124.

[44] C. D. Shaffer, 1997; West Mifflin, Pennsylvania.

[45] Z. Wang, D. Orejon, K. Sefianec, Y. Takata, Phys. Chem. Chem. Phys., 2019, 21. 